\title{
Topological Graphene Imaging and Fabrication of Devices
}

\section{Citation}

Bell, David C., Wei Li Wang, Sagar Bhandari, Robert Westervelt, and Efthimios Kaxiras. 2012. Topological graphene imaging and fabrication of devices. Microscopy and Microanalysis 18(Supplement S2): 1534-35.

\section{Published Version}

doi:10.1017/S143192761200952X

\section{Permanent link}

http://nrs.harvard.edu/urn-3:HUL.InstRepos:10609627

\section{Terms of Use}

This article was downloaded from Harvard University's DASH repository, and is made available under the terms and conditions applicable to Open Access Policy Articles, as set forth at http:// nrs.harvard.edu/urn-3:HUL.InstRepos:dash.current.terms-of-use\#OAP

\section{Share Your Story}

The Harvard community has made this article openly available.

Please share how this access benefits you. Submit a story.

Accessibility 


\title{
Topological Graphene Imaging and Fabrication of Devices
}

\author{
David C. Bell ${ }^{1,3}$, Wei Li Wang ${ }^{1}$, Sagar Bhandari ${ }^{1}$, Robert Westervelt ${ }^{1,2}$ and Efthimios Kaxiras ${ }^{1,2}$ \\ ${ }^{1}$ School of Engineering and Applied Science, Harvard University, Cambridge, MA U.S.A. \\ ${ }^{2}$ Physics Department, Harvard University, Cambridge, MA U.S.A. \\ ${ }^{3}$ Centre for Nanoscale Systems, Harvard University, Cambridge MA U.S.A.
}

A major challenge researchers face is to understand how electrons move through nanoscale graphene structures. The carriers are quite unlike those in conventional semiconductors or metals. The electrons and holes are chiral particles that pass through potential barriers easily (the Klein paradox) and the edges of graphene reconstruct and create new electron states. [1] Graphene is promising for atomic scale devices - to make and understand these structures we need nanoscale probes. We have addressed this challenge directly by developing a process for fabricating (Fig. 1) and imaging ballistic electron flow in graphene (Fig. 2) and controlling graphene quantum dots using cooled SPM instruments and techniques adapted from our earlier work [2].

Our current research fabricates graphene structures with nanosculpting and imaging using TEM, STEM and Helium Ion Microscopy. Using the Helium Ion and electron beams it is possible to etch and mill graphene to fabricate structures such as ribbons and quantum point contact structures [4]. The Helium Ion Microscope operates like a scanning electron microscope, except that the image is provided by a scanned He ion beam, providing new contrast mechanisms and a novel way to etch "soft" materials (Fig. 1) [5]. The quantum point contact (QPC) sample size scales (Fig. 1) $(\sim 10-100 \mathrm{~nm})$ are large enough to transfer between the STEM and SPM for electron flow measurements (Fig. 2). Our second phase is toward the atomic scale - done directly inside a Zeiss Libra aberration-corrected STEM by using the electron beam to both cut and image graphene structures. A review of current progress will be presented.

We also show direct real-space images of the ripples in a few-layer graphene (FLG) membrane resolved at the atomic scale using monochromated aberration-corrected transmission electron microscopy (TEM). The thickness of FLG amplifies the weak local effects of the ripples, resulting in spatially-varying TEM contrast that is unique up to inversion symmetry. We compare the characteristic TEM contrast with simulated images based on accurate first-principles calculations of the scattering potential (Fig. 3). Our results characterize the ripples in real space, and suggest that such features are likely common in ultra-thin materials, even in the nanometer-thickness range [3].

\section{References}

[1] Kyoko Nakada, Mitsutaka Fujita, Gene Dresselhaus and Mildred S. Dresselhaus, "Edge state in graphene ribbons: Nanometer size effect and edge shape dependence," Phys Rev B 54, 17954 (1996).

[2] J. Berezovsky, M.F. Borunda, E.J. Heller and R.M. Westervelt, "Imaging Coherent Transport in Graphene (Part I): Mapping Universal Conductance Fluctuations," Nanotechnology 21, 274013 (2010); " Imaging Coherent Transport in Graphene (Part II): Probing Weak Localization," Nanotechnology 21, 274014 (2010).

[3] Wei L. Wang, Sagar Bhandari, Wei Yi, David C. Bell, Robert Westervelt, Efthimios Kaxiras, "Direct imaging of atomic-scale ripples in few-layer graphene", In Publication.

[4] David C. Bell, Max C. Lemme, Lewis A. Stern, Jimmy R. Williams, Charles M. Marcus, "Precision cutting and patterning of graphene with helium ions", Nanotechnology 20, 455301 (2009).

[5] David C. Bell "Contrast Mechanisms and Image Formation in Helium Ion Microscopy", Microscopy and Microanalysis, 15 (2009), pp. 147-153. 

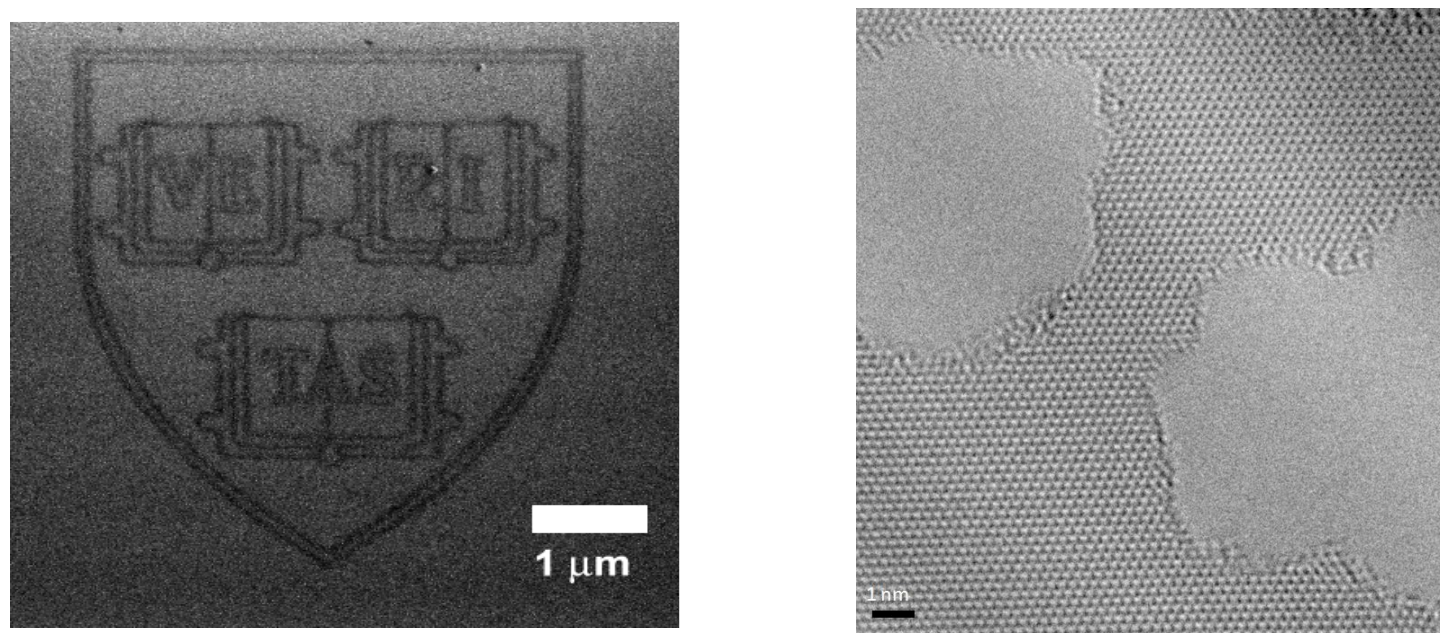

Figure 1. Left, Helium ion beam etching of a graphene sheet. Right, Uncontaminated nano-scale graphene (FLG) junction formed in TEM (Libra) by sculpting.

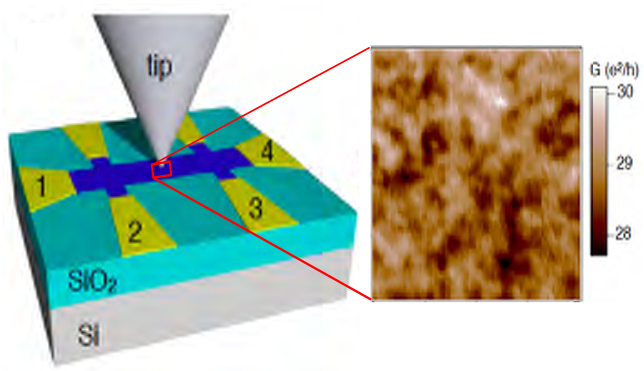

Figure 2. Left, SPM tip above a graphene Hall bar sample; the conducting tip creates a movable scatterer in the 2DEG below. ${ }^{2}$ Right, SPM image of universal conductance fluctuations at $4 \mathrm{~K}$.

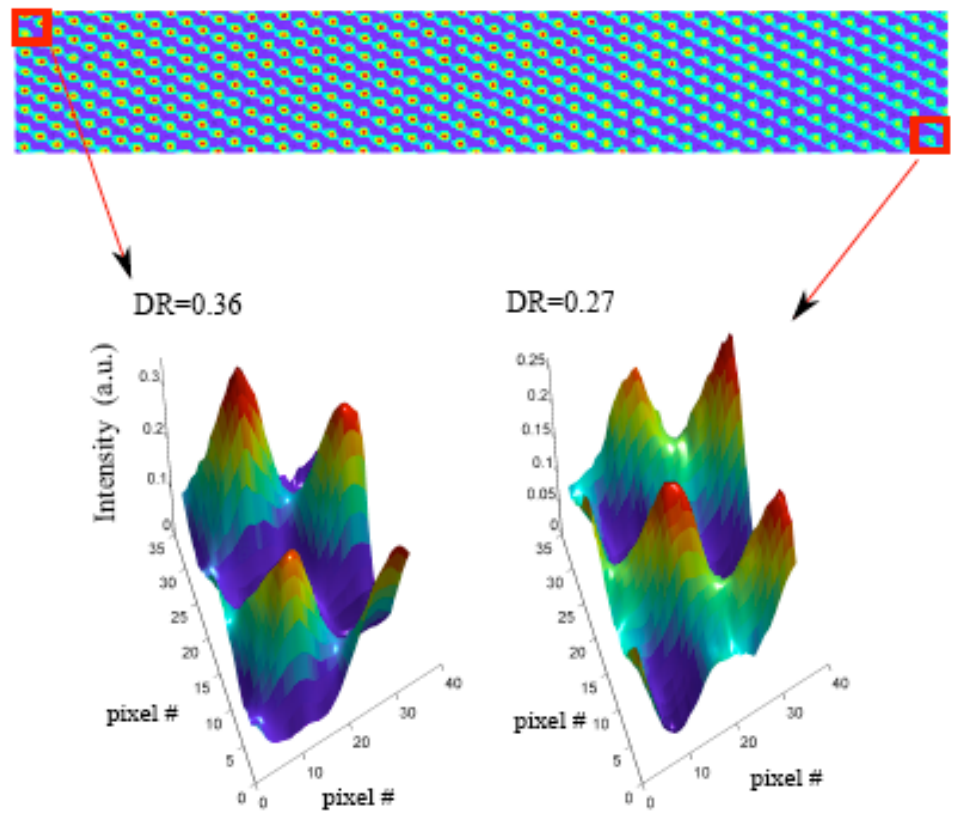

Figure 3. Fine details in TEM contrast and variation of image dynamic range. An image with pixel size of 32 by 32 is taken at each end of the image in Fig. 1e. Three-dimensional representation of the intensity is plotted for two small regions, for which the normalized dynamic range is 0.36 and 0.27 respectively. 